\title{
Improving Strategies to Assess Competitive Effects of Barred Owls on Northern Spotted Owls in the Pacific Northwest
}

A male barred owl (Strix varia) hears the call of
another owl during the night. If the call is from
another barred owl, he might respond with a
series of rapid hoots to inform the intruder of his presence. If the call is from a northern spotted owl (Strix occidentalis caurina) ${ }^{1}$, however, he may fly swiftly and silently towards the sound to get a closer look. When the spotted owl call is broadcast by researchers performing a routine survey of spotted owls, and the barred owl sees that there are no owls present, he may remain silent and watch. Barred owls typically are shy around people, and a quiet owl is less likely to be seen by researchers in the dark forest. As a result, it is often difficult to document the occurrence of barred owls during surveys in which researchers use spotted owl calls.

A scientific study has determined that survey methods designed for spotted owls do not always detect barred owls that are actually present in spotted owl habitat. The researchers suggest that strategies to address potential interactions between spotted owls and barred owls will require carefully designed surveys that account for response behaviors and imperfect detection of both species. Species-specific sampling methods, which are proposed, can be used by forest managers to determine the occurrence and distribution of barred owls with high confidence. This fact sheet provides highlights of the research (Wiens and others, 2011).

\section{Background}

Under the Endangered Species Act, resource managers are charged with developing research and management strategies that aid in the recovery of the northern spotted owl. Current strategies rely on the preservation of structurally complex forest habitat, but declines in spotted owl populations also may be a consequence of increased competition for space, habitat, and food with invading barred owls.

\footnotetext{
${ }^{1}$ There are three subspecies of spotted owl: northern (S. o. caurina), California (S. o. occidentalis), and Mexican (S. o. lucida). This overview relates primarily to the northern spotted owl, hereafter referred to as spotted owl. This subspecies is listed as "Threatened" under the Endangered Species Act.
}

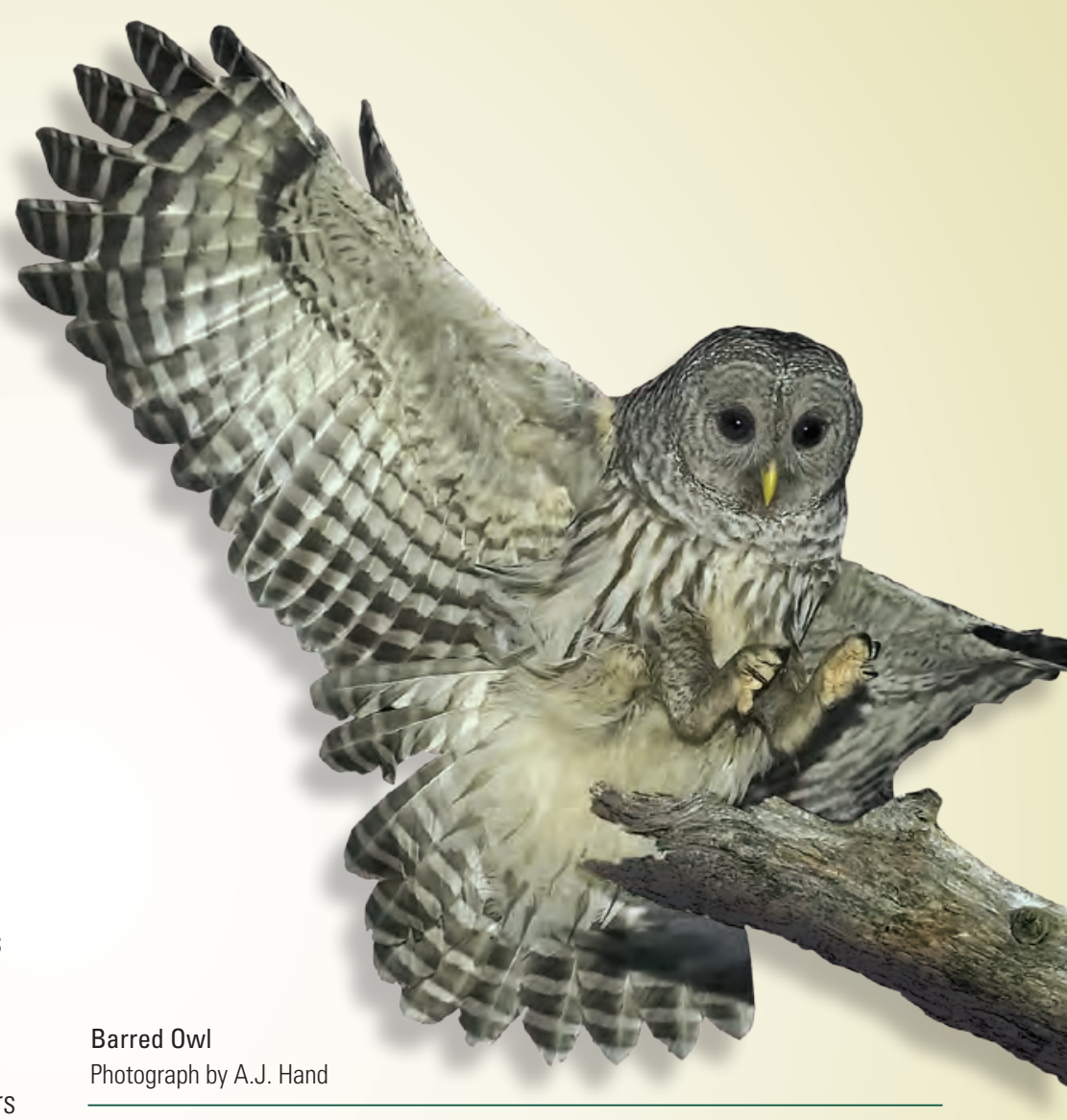

Evidence of barred owls expanding their range into western forests is based on incidental detections of barred owls documented during surveys of spotted owls. In response to a growing concern among land managers about the effects of invading barred owls on spotted owls, eight Federal and State agencies jointly funded a comprehensive 4-year study that specifically examined the competitive interactions between both owl species. At the onset of that study, researchers needed to be confident that they were using survey methods that worked equally well for both species. Survey protocols for spotted owls are well documented and tested, and regularly conducted associated with population monitoring objectives. Survey protocols for barred owls, however, are recently developed and have not been fully tested in the field. As a consequence, the first objectives of the larger study of competitive interactions were to investigate the effectiveness of the two survey protocols in detecting barred owls and to determine the occurrence of barred owls independently of spotted owl monitoring efforts. 
